\title{
Pruritic cutaneous lesions mimicking dermatitis artefacta in the course of mediastinal gray-zone lymphoma
}

\author{
Karolina Englert, Agata D. Kłosowicz, Barbara Grześkowiak, Anna Wojas-Pelc
}

Department of Dermatology, University Hospital, Krakow, Poland

Adv Dermatol Allergol 2020; XXXVII (2): 280-282

DOI: https://doi.org/10.5114/ada.2020.94850

Unlike nodular sclerosis Hodgkin lymphoma (NSHL) and primary mediastinal B-cell lymphoma ( $\mathrm{PMBL}$ ) that are common types of lymphomas present in the mediastinum, mediastinal gray-zone lymphoma (MGZL) is extremely rare [1]. These tumours are called "gray zone" because of their overlapping morphological and immunophenotypic features [2]. In the case of MGZL, the surface immunoglobulin is not expressed, there is at least one B-cell marker expressed and the presence of other cell surface markers varies. These tumours can exhibit prominent expression of CD20, CD79a with a weaker or absent expression of CD15 and CD30 or vice versa. The minimum immunohistochemical panel, essential for establishing a correct diagnosis, includes CD20, PAX5, MUM1, CD30, CD15, and EBV by in situ hybridization. MGZL may present in one patient the PMBL-like morphology and NSHLlike immunophenotype or vice versa [3, 4]. Historically, these tumours were classified as "anaplastic large-cell lymphoma Hodgkin's-like" [5].

MGZLs predominantly affect young men. MGZL is often present as a single mass in the mediastinum that grows to large sizes [6]. As a consequence it can cause chest pain, breath difficulties, fatigue or weight loss. The aetiology has not been well defined, although certain genetic mutations have been implicated. In recently published studies, the epigenetic profiles of these neoplasms are suggested to be crucial for the confirmation of diagnosis [3, 7]. According to Eberle et al., prediction of MGZL can be made on the basis of DNA methylation profiles (including such genes as HOXA5, MMP9, EPHA7, DAPK1) [7]. Their rarity and recent identification have led to uncertainty regarding the therapeutic strategy, clinical characteristics and treatment. NSHL and PMB are treated differently; therefore, the optimal treatment for gray zone lymphoma is unclear [8]. The condition is reported to have a poorer prognosis than both primary mediastinal large B-cell lymphoma and classical Hodgkin's lymphoma [9].

To our knowledge, this is the first report about cutaneous lesions in the course of MGZL which were treated as dermatitis artefacta.
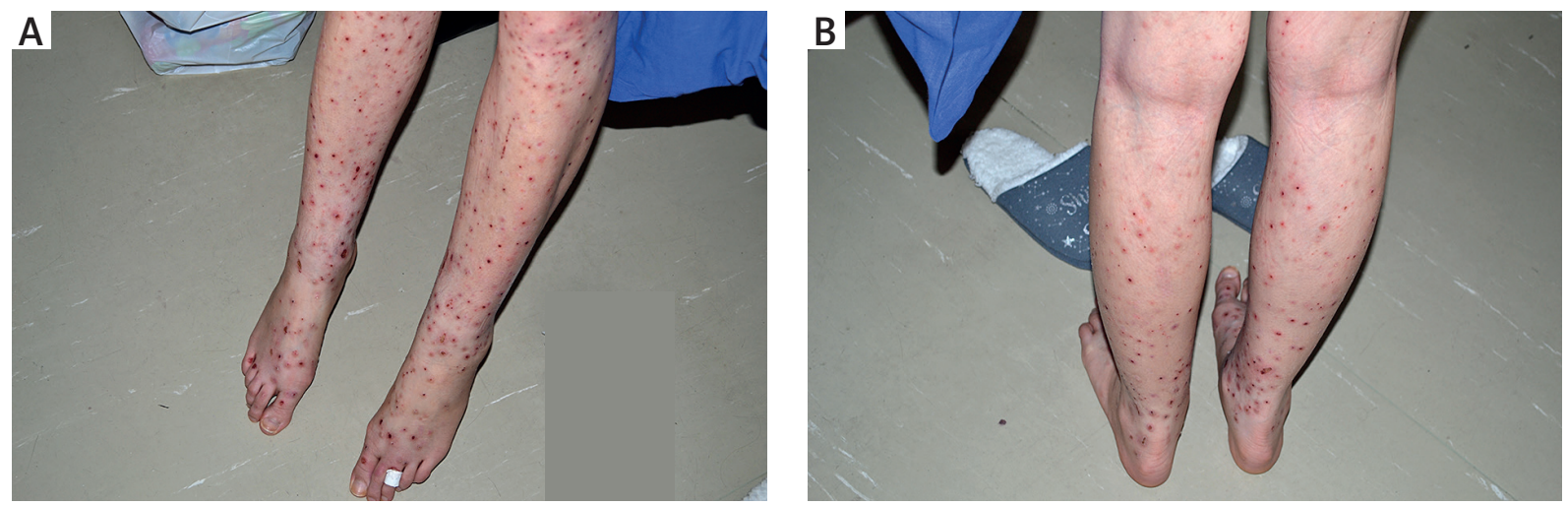

Figure 1. Disseminated erythematous papules and superficial erosions covered with crusts on the lower extremities

Address for correspondence: Karolina Englert MD, Department of Dermatology, Jagiellonian University Hospital, Krakow, Poland, phone: +48 600248 916, e-mail: englertderm@gmail.com Received: 21.10.2018, accepted: 17.01.2019. 


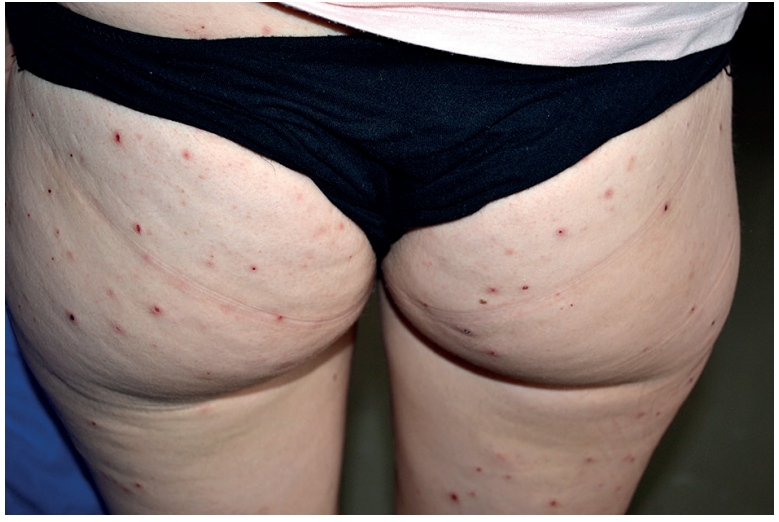

Figure 2. Disseminated erythematous papules and superficial erosions covered with crusts on the buttocks
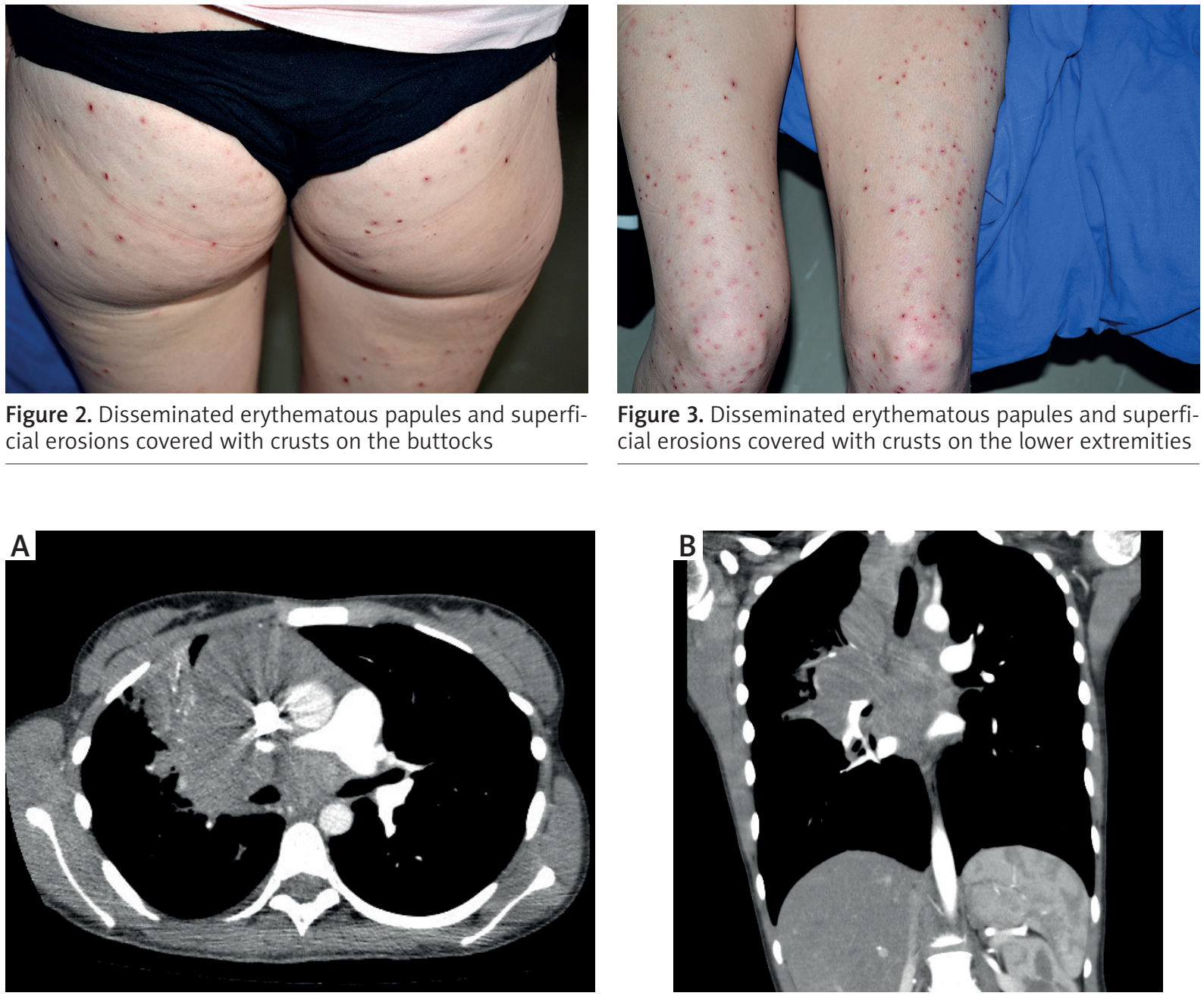

Figure 3. Disseminated erythematous papules and superficial erosions covered with crusts on the lower extremities

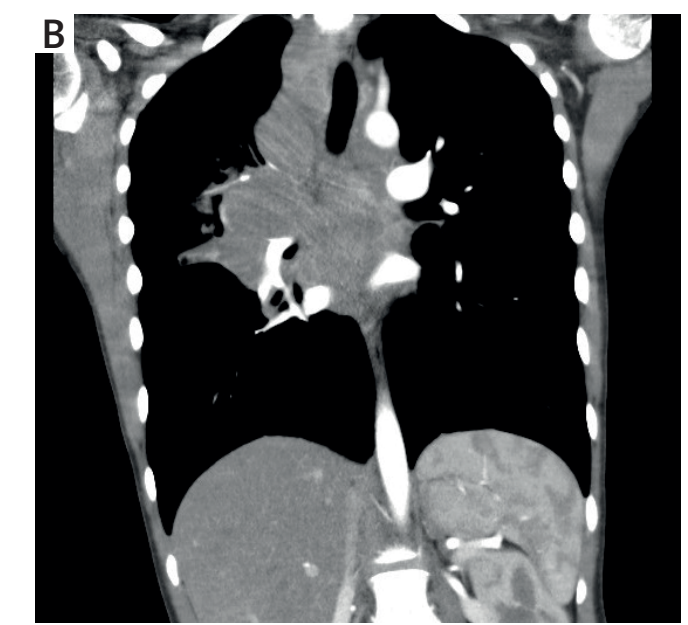

Figure 4. Mediastinal gray-zone lymphoma in an 18-year-old woman presented with the eruption of erythematous papules on the lower and upper extremities. Axial (A) and coronal CT (B) images show the extensive, mediastinal nodular mass with paratracheal, tracheal bifurcation and right hilar lymphadenopathies. Thanks to courtesy of the Radiology Department of the Jagiellonian University in Krakow (Head of Department Wiesław Pawlik, MD, PhD)

A 19-year-old woman presented with a 6-month history of intense pruritus followed by the eruption of erythematous papules on the lower and upper extremities (Figures 1-3). Pruritus exacerbated at night (after warming up of the body) and after a hot bath.

About 1 month after the onset of the symptoms, the patient began to have erythematous papules on the lower legs and buttocks and sequentially on the upper extremities. The patient was scratching the lesions, which resulted in the presentation of superficial erosions covered with crusts. There was no history of fever or weight loss but the patient complained of severe tiredness and night sweats. A clinical examination did not reveal any adenopathy or hepatosplenomegaly. A skin biopsy showed epidermal acanthosis, perivascular infiltrate of lymphocytes and histiocytes in the dermis. The histopathological examination was not characteristic but it could develop secondary to trauma.
Having no clinical improvement of the lesions we decided to extend our diagnostic regimen and performed laboratory tests and image explorations. The laboratory test results were as follows: IgE rate: $17.2 \mathrm{IU} / \mathrm{ml}$ (normal $<100$ ), C-reactive protein: $86.6 \mathrm{mg} / \mathrm{l}$ (normal < 5), leukocytes: $15.74 . G / l$ (normal 4-10) with higher rates of neutrophils $(13.44 \mathrm{G} / \mathrm{l})$ and monocytes $(0.96 \mathrm{G} / \mathrm{l})$. Protein electrophoresis showed total body depletion of protein: 61.7 g/l (normal 66-87) and albumin: 28.51 g/l (normal 35-55); increased intensity of $\alpha 1$ : $3.89 \mathrm{~g} / \mathrm{l}$ (normal 0.9-2.1) and $\alpha 2$ : 10.12 (normal 5-7.9) band and slightly increased intensity of $\gamma$-globulin band: 11.85 (6.5-11.5). Serologic tests ruled out hepatitis B and C, HIV, and tuberculosis infection. Lactate dehydrogenase (LDH) and 2-microglobulin were within normal levels. Anti-tissue transglutaminase antibodies IgA, IgG, antiendomysial antibodies IgA, IgG as well as ANA antibodies were negative. The ultrasound examination of the abdomen and pelvis did not reveal any abnormalities. 
Chest $X$-rays have revealed a mediastinal nodular mass, an oval shade over the diaphragm on the right side, a circular shade in the lower region of the right lung and a slight amount of right-sided pleural effusion. Computed tomography $(\mathrm{CT})$ scan confirmed the presence of the extensive, mediastinal nodular mass with anterior, middle mediastinal and right hilar lymphadenopathies with several nodules and consolidations in the right lung and to a lesser extent in the left lung. The mass surrounded superior vena cava (triggering its stenosis), ascending aorta, right pulmonary artery, right superior pulmonary vein and pericardium (Figures 4 A, B).

A lymph node biopsy revealed a polymorphic cellular infiltrate (lymphocytes TCD3 positive, B CD20 positive; histiocytes, eosinophils) with predominant infiltrate of the HRS (Hodgkin/Reed-Stenberg) cells. The lymphoma displayed many features consistent with Hodgkin lymphoma. Nevertheless the significant expression of B-cell markers led to the diagnosis of unclassifiable cell lymphoma, with features intermediate between Hodgkin lymphoma and diffuse large B-cell lymphoma (Mediastinal gray-zone lymphoma).

The chemotherapy was initiated (6 cycles of etoposide - cyclophosphamide-hydroxydaunomycin-vincristineprednisone, E-CHOP). Subsequently, involved-field radiation therapy (IFRT) was performed (36 Gy/18 fraction; breathhold intensity modulated radiotherapy, IMRT). The patient achieved a complete remission of pruritic skin lesions and the mediastinal tumour.

To our knowledge, this is the first case report of generalized pruritic rash as a paraneoplastic symptom heralding the diagnosis of MGZL.

Chronic pruritus is defined as an itch lasting more than 6 weeks [10]. Various studies have linked generalized pruritus and pruritic rash to internal malignancy, in particular lymphoproliferative disease [11]. Paraneoplastic pruritus develops before a clinically evident cancer, is not caused by the direct effect of the tumour and resolves after treatment [12]. Due to the rarity of MGZL, the cutaneous manifestations of this condition have been poorly studied. Taking into consideration the fact that the features overlap DLBCL and $\mathrm{CHL}$, we highlighted the most common malignancy associated with generalized pruritus, i.e. HD. A number of studies have reported a significant incidence of generalized pruritic rash in Hodgkin's disease, even up to $25 \%$ [12]. As it has been associated with a poor prognosis some authors proposed to include generalized pruritic rash as a B type symptom [13]. Rash that is generalized is most often due to paraneoplastic manifestation and precedes other clinical signs. It often resolves with treatment of Hodgkin's disease [12]. A differential diagnosis of generalized rash accompanied by severe pruritus includes contact dermatitis, insect bites, lichen planus, scabies or nodular prurigo. In our case, clinical, dermoscopic and histopathological findings did not confirm either of these conditions. The plausible explanation of the rash in the patient is paraneoplastic manifestation considering the generalized nature, the maculopapular presentation resolv- ing after the course of chemotherapy and the skin biopsy which ruled out cutaneous spread of the tumour.

This case highlights the importance of a good systemic examination and considering systemic causes like mediastinum lymphomas as a possible cause of generalized pruritic papular rash if it is not resolving with usual treatment. The diagnosis of dermatitis artefacta can be established only after a careful process of ruling out other not cutaneous and systemic conditions.

\section{Acknowledgments}

The study was conducted at the Jagiellonian University Hospital in Krakow, Poland, Department of Dermatology.

\section{Conflict of interest}

The authors declare no conflict of interest.

\section{References}

1. Swerdlow SH, Campo E, Harris NL, et al. WHO classification of tumors of haematopoietic and lymphoid tissues. $4^{\text {th }}$ ed. Lyon: International Agency for Research on Cancer 2008.

2. Traverse-Glehen A, Pittaluga S, Gaulard P, et al. Mediastinal gray zone lymphoma: the missing link between classic Hodgkin's lymphoma and mediastinal large B-cell lymphoma. Am J Surg Pathol 2005; 29: 1411-21.

3. Eberle FC, Salaverria I, Steidl C, et al. Gray zone lymphoma: chromosomal aberrations with immunophenotypic and clinical correlations. Mod Pathol 2011; 24: 1586-97.

4. Pilichowska M, Pittaluga S, Ferry JA, et al. Clinicopathologic consensus study of gray zone lymphoma with features intermediate between DLBCL and classical HL Blood Adv 2017; 1: 2600-9.

5. Pileri S, Bocchia M, Baroni CD, et al. Anaplastic large cell lymphoma (CD30+/Ki-1+): results of a prospective clinico-pathological study of 69 cases. Br J Haematol 1994; 86: 513-23.

6. Quintanilla-Martinez L, Fend F. Mediastinal gray zone lymphoma. Haematologica 2011; 96: 496-9.

7. Eberle FC, Rodriguez-Canales J, Wei L, et al. Methylation profiling of mediastinal gray zone lymphoma reveals a distinctive signature with elements shared by classical Hodgkin's lymphoma and primary mediastinal large B-cell lymphoma. Haematologica 2011; 96: 558-66.

8. Dunleavy K, Grant C, Eberle FC, et al. Gray zone lymphoma: better treated like Hodgkin lymphoma or mediastinal large B-cell lymphoma. Curr Hematol Malig Rep 2012; 7: 241-7.

9. Dunleavy K, Bollard CM. Sobering realities of surviving Hodgkin lymphoma. Blood 2011; 117: 1772-3.

10. Yosipovitch G, Greaves MW. Definitions of itch. In: Itch: Basic Mechanisms and Therapy. Yosipovitch G, Greaves MW, Fleischer AB, McGlone F (eds.). Marcel Dekker, New York 2004; 2.

11. Wang $\mathrm{H}$, Yosipovitch $\mathrm{G}$. New insights into the pathophysiology of chronic itch in patients with end stage renal failure, chronic liver disease and lymphoma. Int J Dermatol 2010; 49: 1-11.

12. Yosipovitch G. Chronic pruritus: a paraneoplastic sign. Dermatol Ther 2010; 23: 590-6.

13. Gobbi PG, Attardo-Parrinello G, Lattanzio G, et al. Severe pruritus should be a B-symptom in Hodgkin's disease. Cancer 1983; 51: 1934-6. 